\title{
La teneur en acides gras polyinsaturés du lait maternel : un marqueur biologique fiable du niveau de consommation des populations
}

\author{
Philippe GUESNET ${ }^{1}$ \\ Nicole $\mathrm{COMBE}^{2}$ \\ Gérard AILHAUD ${ }^{3}$ \\ Jean-Marc ALESSANDRI ${ }^{1}$ \\ ${ }^{1}$ NuRéLiCe, UR909, INRA, \\ F 78352 Jouy-en-Josas, France \\ <Philippe.Guesnet@jouy.inra.fr> \\ 2 ITERG, Nutrition Métabolisme \& Santé, \\ Université Bordeaux 1, \\ avenue des Facultés, 33405 Talence Cedex \\ 3 ISDBC, \\ Université de Nice Sophia-Antipolis, CNRS, \\ 28 avenue Valrose, \\ Nice 06100, France
}

\begin{abstract}
Polyunsaturated fatty acids (PUFA) are nutritionally important constituents of breast milk to support normal growth, immune function and central nervous system development of newborn infants. Both linoleic acid (18:2 n-6; LA) and alpha-linolenic acid (18:3n-3;ALA), the essential fatty acids, precursors of n- 6 and n-3 long-chain PUFA. LA and LNA contents in human milk reflect differences in dietary fats consumed by the mothers, including those consumed during several months (long term impact). The composition of breast milk from this point of view is a reliable biological marker of the level of habitual consumption of PUFAs in different populations. An increase of LA content for the 1950-1990 period, without any change of LNA content, has been reported in breast milk of women living in western countries, reflecting changes in LA intake by the mother.
\end{abstract}

Key words: human milk, poly unsaturated fatty acid, consumption of PUFAs
Le lait humain contient environ $40 \mathrm{~g}$ de lipides totaux par litre, qui se présentent à plus de $98 \%$ sous la forme de triglycérides [1]. Les triglycérides du lait de femme constituent plus de la moitié de l'énergie calorique et jouent donc un rôle vital pour la croissance et le développement du nourrisson. Le lait maternel apporte également des acides gras polyinsaturés (AGPI) dont le rôle sur la croissance pondérale et le développement neurosensoriel du nourrisson est établi [2]. Ces AGPI sont représentés par les précurseurs essentiels des séries n-6 et n-3 (obligatoirement apportés par l'alimentation), respectivement les acides linoléique (18:2n-6) et $\alpha$-linolénique (18:3n-3), et leurs dérivés à longue chaîne, parmi lesquels on considère que deux sont indispensables au développement postnatal: l'acide arachidonique (20:4n-6) pour la série $n-6$ et l'acide docosahexaénoïque (22:6n-3) pour la série $n-3$ [2]. Dans les pays occidentaux, $15 \%$ en moyenne des acides gras totaux du lait de femme sont des AGPI, dont I'acide linoléique (à plus de $80 \%$ ), I'acide $\alpha$-linolénique (6 à $7 \%$ ), et pour la part restante, les dérivés à longue chaîne (AGPI-LC) dont les représentants majeurs sont I'acide arachidonique et l'acide docosahexaénoïque (tableau 1) [3].

La teneur en AGPI du lait maternel évolue en fonction de plusieurs facteurs : nutritionnels car elle est directement dépendante des apports dans I'alimentation maternelle, physiologique, car elle évolue avec l'âge gestationnel et la durée de la lactation, et même géographiques car elle varie d'une population à une autre (tableau 1). Les analyses du lait de femmes françaises que nous avons réalisées dans les années 1990 ont révélé une dispersion importante de la teneur en 18:2n-6 dans le lait mature (min.-max. : 6,1-22,7 \%) qui était à l'origine de la variabilité individuelle encore plus importante de la valeur du rapport 18:2n-6/18:3n-3 (min.-max. : 5-47) [4, 5]. Par ailleurs, $20 \%$ de la population étudiée présentaient un déséquilibre chronique de ce rapport qui se maintenait à une valeur élevée tout au long de la lactation (18:2n-6/18:3n-3 > 25), reflétant une consommation alimentaire habituelle trop élevée d'acide linoléique souvent associée à une consommation trop faible d'acide $\alpha$-linolénique. La relation entre la consommation $d^{\prime} A G P I$ et leurs teneurs dans le lait maternel a également été mise en évidence pour les acides arachidonique et docosahexaénoïque $[3,6]$. Cependant, la relation entre la composition en acides gras des aliments et celle du lait maternel n'est pas directe, car elle dépend étroitement du métabolisme spécifique de chaque famille $d^{\prime}$ acides gras. Ainsi, pour un même niveau $d^{\prime}$ apport alimentaire en acide $\alpha$-linolénique et en acide linoléique, la teneur en acide $\alpha$-linolénique du lait maternel est moindre que celle de l'acide linoléique et le rapport

Tableau 1. Teneurs moyennes, minimales et maximales (\% des acides gras totaux) rencontrées dans le lait mature de femmes vivant dans les pays occidentaux (adapté de [3])

\begin{tabular}{|c|c|c|c|c|c|c|}
\hline & $18: 2 n-6$ & $20: 4 n-6$ & $18: 3 n-3$ & $22: 6 n-3$ & $\begin{array}{l}18: 2 n-6 / \\
18: 3 n-3\end{array}$ & $\begin{array}{l}20: 4 n-6 / \\
22: 6 n-3\end{array}$ \\
\hline Moyenne & 10 à 16 & 0,40 à 0,54 & 0,58 à 1,44 & 0,19 à 0,42 & 7,3 à 22,1 & 1,12 à 2,61 \\
\hline Minimal & 7,5 & 0,15 & 0,28 & 0,09 & 4,5 & 0,55 \\
\hline Maximal & 20,2 & 1,50 & 2,04 & 0,97 & 58,2 & 15,0 \\
\hline
\end{tabular}

Le lait maternel renferme également divers intermédiaires de la voie de métabolisation des AGPI : série n-6 (environ 0,7 \% des acides gras totaux), 18:3n-6, 20:2n-6, 20:3n-6 ; série n-3 (environ 0,5 \%), 18:4n-3, 20:5n-3, 22:5n-3. II contient en moyenne environ $50 \%$ d'acides gras saturés et $35 \%$ d'acides gras mono-insaturés. 
18:2n-6/18:3n-3 s'en trouve augmenté d'un facteur 2 par rapport à celui de l'aliment (figure 1) [7]. Le catabolisme oxydatif plus élevé de l'acide $\alpha$-linolénique par rapport à celui de l'acide linoléique peut expliquer cette amplification du rapport dans le lait [8].
Nous disposons de peu de données expérimentales sur la provenance métabolique des AGPI composant les acides gras du lait maternel (alimentation, bioconversion par le foie, mobilisation des réserves corporelles). Il apparaît toutefois que la teneur en acide linoléique du lait est

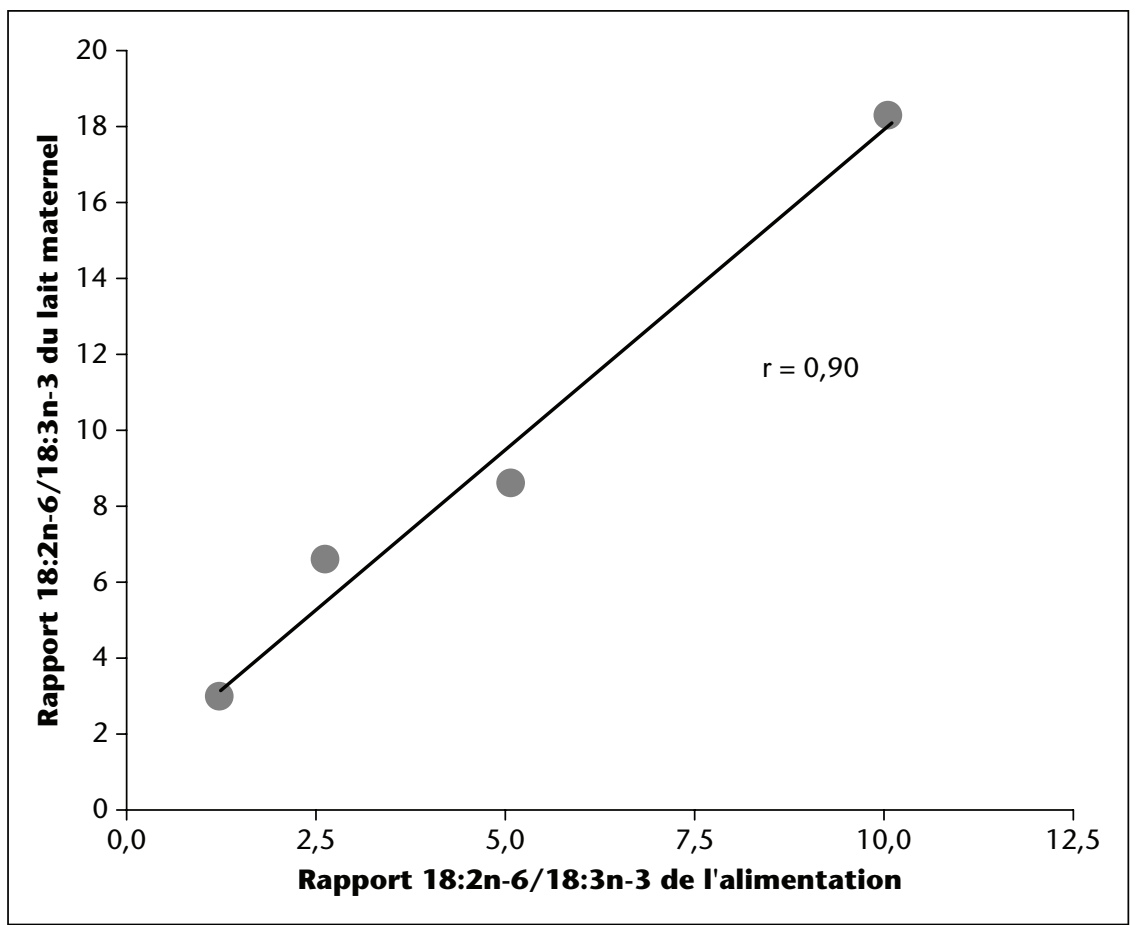

Figure 1. Corrélation entre le rapport acide linoléique (18:2n-6)/acide $\alpha$-linolénique (18:3n-3) de I'alimentation maternelle et celui du lait maternel (adapté de [7]).

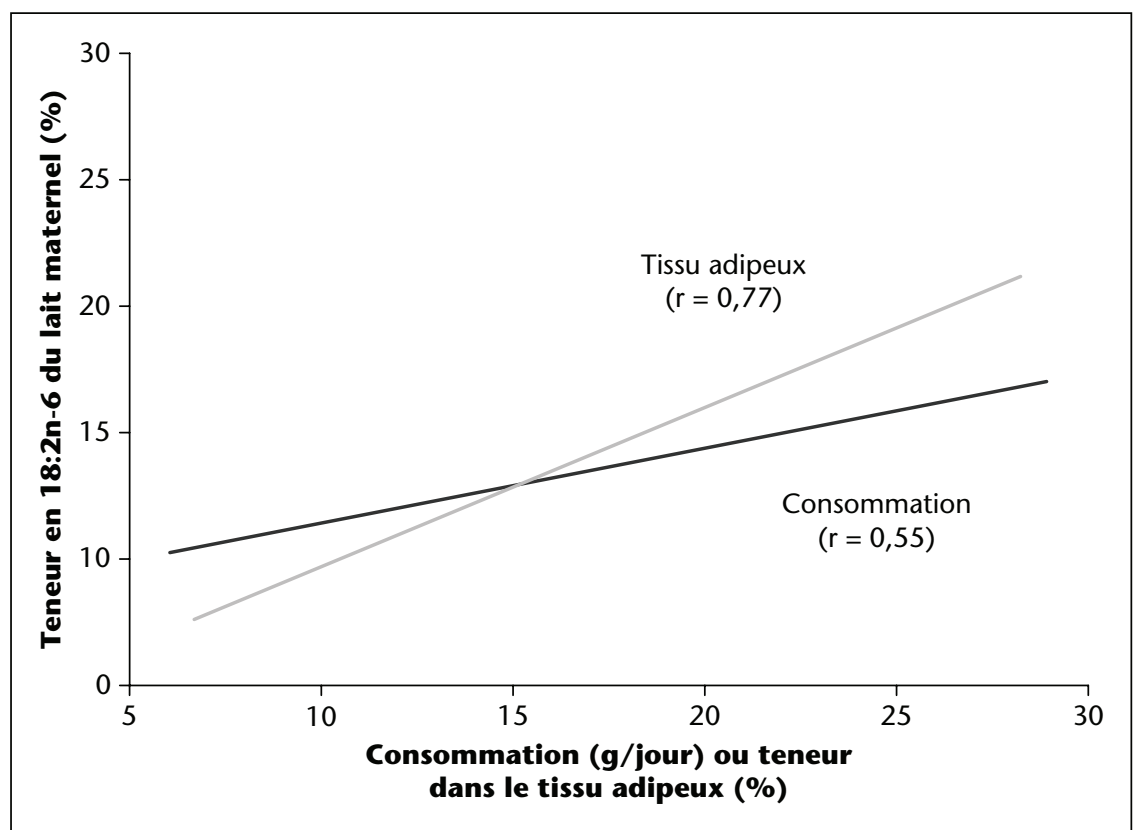

Figure 2. Corrélation entre la teneur en acide linoléique (18:2n-6) du lait maternel et la consommation (g/jour) ou la teneur du tissu adipeux en acide linoléique de la femme allaitante (adapté de [9]).

plus étroitement liée à la teneur du tissu adipeux qu'elle ne l'est à celle de l'alimentation (figure 2) [9]. L'ingestion par des femmes allaitantes d'un bolus d'acide linoléique marqué au carbone 13, suivie de l'analyse de la composition et du niveau d'enrichissement isotopique de leurs laits, a permis de montrer qu'un tiers de cet acide gras du lait provient directement de I'alimentation (reflet de l'aliment sur le court terme) et que les $2 / 3$ restants sont issus de la mobilisation du tissu adipeux (reflet de l'alimentation sur le long terme) [10]. Ainsi, les variations de teneur en AGPI observées dans les différents laits maternels reflètent des différences de consommation accumulées sur plusieurs mois. La composition du lait maternel est donc un marqueur biologique fiable du niveau de consommation habituelle des AGPI par les différentes populations.

En nous appuyant sur ce constat, nous avons récemment évalué les changements qualitatifs des apports alimentaires en AGPI, concernant notamment I'équilibre entre $n-6$ et $n-3$, dans I'alimentation humaine au cours de ces dernières décennies dans les pays industrialisés [3]. En ce qui concerne les USA, pays pour lequel on dispose de données longitudinales solides, il apparaît que la teneur en acide linoléique s'est accrue au cours de la période 1960-1980, avoisinant les 6-7\% des acides gras totaux au début de cette période pour atteindre une valeur maximale de $15-16 \%$ dans les années 1980 (figure 3) [3]. Pendant la même période, la teneur en acide $\alpha$-linolénique est restée stable, se traduisant par l'accroissement progressif du rapport $n-6 / n-3$, lequel peut aujourd'hui atteindre des valeurs supérieures à 20 alors que le niveau correspondant à une alimentation maternelle équilibrée (rapport de 5) est estimé autour de 10 (figure 1) [3]. Cette évolution temporelle est observée dans la majorité des pays industrialisés, mais avec une moindre amplitude et un décalage dans le temps par rapport aux USA. Ces modifications de consommation proviennent de changements profonds d'habitudes alimentaires, impliquant la consommation de matières grasses visibles (consommation d'huiles alimentaires riches en acide linoléique et pauvres en acide $\alpha$-linolénique) et cachées (matières grasses animales dont la composition a évolué) [3]. Pour l'acide linoléique, cette évolution a été également identifiée dans les études de consommation basée sur le niveau d'utilisation des huiles végétales dans l'alimentation humaine (figure 3) [3].

En conclusion, I'évaluation du niveau de consommation AGPI des populations, au travers de l'analyse de la composition en acides gras du lait de femme, s'avère une approche expérimentale originale et plus précise qu'une estimation de la consommation effectuée à 


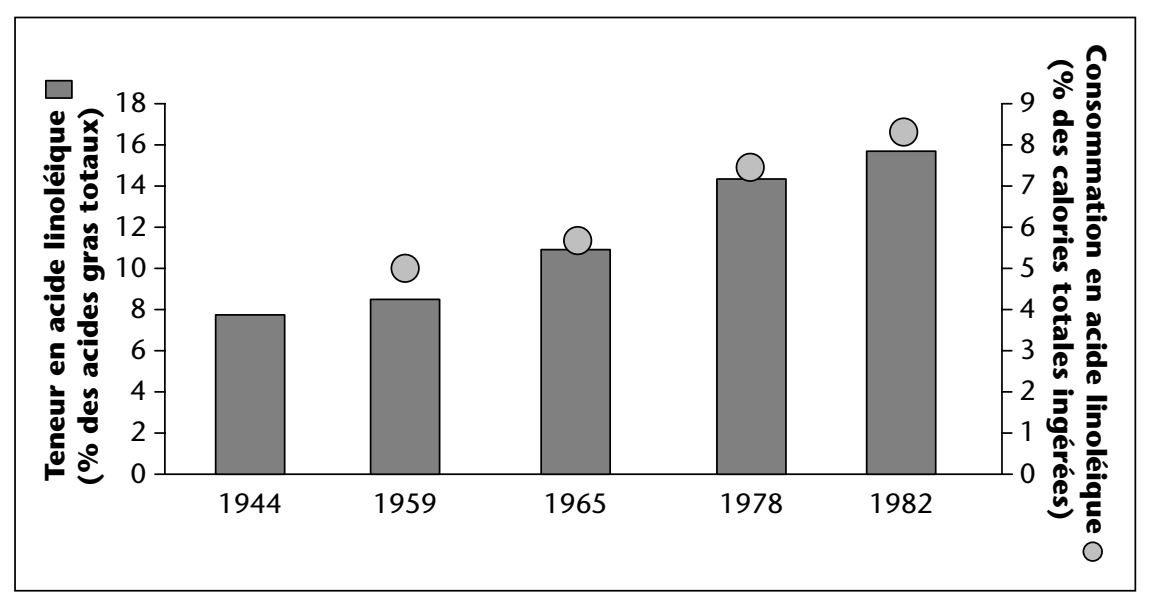

Figure 3. Évolution de la consommation en acide linoléique aux États-Unis entre les années 1950 et 1980 - Estimation basée d'après la teneur du lait maternel et le niveau de consommation apparente (adapté de [3]).

partir de tables alimentaires préétablies [3]. L'étude multicentrique qui est publiée dans ce fascicule illustre parfaitement l'intérêt nutritionnel de cette approche épidémiologique dont l'objectif majeur était de déterminer si l'augmentation de l'offre proposée à la consommation d'acide $\alpha$-linolénique depuis les années 1990, avait eu un impact réel sur le statut nutritionnel des français. Les données recueillies permettent de situer le niveau actuel de consommation quotidienne en acide $\alpha$ linolénique de la femme allaitante autour de $1,2 \mathrm{~g}$, ce qui reste encore insuffisant en regard des besoins $(2 \mathrm{~g})$. La tendance à la baisse de la teneur en acide linoléique dans I'alimentation a accompagné cette évolution, contribuant à limiter le déséquilibre entre les AGPI n-6 et n-3. La consommation plus importante d'huiles riches en acide $\alpha$-linolénique sous la forme visible (huile de colza par exemple) et invisible (produits élaborés) a vraisemblablement contribué à améliorer de façon significative le statut en AGPI de la population française.

\section{RÉFÉRENCES}

1. Jensen RG. The lipids of Human milk. CRC Press, Inc, 1989.

2. Guesnet P, Alessandri JM. Acides gras polyinsaturés du lait et développement du système nerveux central du nouveau-né. Cah Nutr Diét $1995 ; 30: 109-16$.

3. Ailhaud G, Massiera F, Weill P, Legrand P, Alessandri J, Guesnet P. Role of dietary fat and n-6 polyunsaturated fatty acids in excessive adipose tissue development. Relationship to childhood obesity. Prog Lipid Res 2006 ; 45 : 203-36.

4. Guesnet $P$, Antoine JM, Rochette De Lempdes JB, Galent A, Durand G. Polyunsaturated fatty acid composition of human milk in France: changes during the course of lactation and regional differences. Eur / Clin Nutr 1993; 47 : 700-10.

5. Guesnet $\mathrm{P}$, Couet $\mathrm{C}$. Alessandri Jm, Antoine Jm, Durand $G$. Variabilité de la teneur en acide linoléique (18:2n-6) et du rapport 18:2n-6/18:3n-3 des lipides dans le lait de femme en France. Ann Ped (Paris) $1995 ; 42: 282-8$.

6. Van Goor SA, Dijck-Brouwer DA, Hadders-Algra $M$, et al. Human milk arachidonic acid and docosahexaenoic acid contents increase following supplementation during pregnancy and lactation. Prostaglandins Leukot Essent Fatty Acids $2009 ; 80: 65-9$.

7. Guesnet P, Alasnier C, Alessandri JM, Durand G. Modifying the $\mathrm{n}-3$ fatty acid content of the maternal diet to determine the requirements of the fetal and suckling rat. Lipids $1997 ; 32$ : 527-34.

8. Poumès-Ballihaut $C$, Langelier $B$, Houlier F. Alessandri Jm, Durand G, Latge C, Guesnet P. Comparative bioavailability of dietary alphalinolenic and docosahexaenoic acids in the growing rat. Lipids $2001 ; 36: 793-800$.

9. Martin JC, Bougnoux P, Fignon A, et al. Dependence of human milk essential fatty acids on adipose stores during lactation. Am / Clin Nutr $1993 ; 58: 653-9$.

10. Demmelmair $H$, Baumheuer $M$, Koletzko B, Dokoupil K, Kratl G. Metabolism of U13Clabeled linoleic acid in lactating women. / Lipid Res 1998 ; 39 : 1389-96. 